\title{
An integrated approach of gene expression and DNA-methylation profiles of WNT signaling genes uncovers novel prognostic markers in Acute Myeloid Leukemia
}

\author{
Erdogan Taskesen ${ }^{1,2^{*}}$, Frank JT Staal ${ }^{3}$, Marcel JT Reinders ${ }^{1,2}$ \\ From The 9th IAPR conference on Pattern Recognition in Bioinformatics \\ Stockholm, Sweden. 21-23 August 2014
}

\begin{abstract}
Background: The wingless-Int (WNT) pathway has an essential role in cell regulation of hematopoietic stem cells (HSC). For Acute Myeloid Leukemia (AML), the malignant counterpart of HSC, currently only a selective number of genes of the WNT pathway are analyzed by using either gene expression or DNA-methylation profiles for the identification of prognostic markers and potential candidate targets for drug therapy. It is known that mRNA expression is controlled by DNA-methylation and that specific patterns can infer the ability to differentiate biological differences, thus a combined analysis using all WNT annotated genes could provide more insight in the WNT signaling.

Approach: We created a computational approach that integrates gene expression and DNA promoter methylation profiles. The approach represents the continuous gene expression and promoter methylation profiles with nine discrete mutually exclusive scenarios. The scenario representation allows for a refinement of patient groups by a more powerful statistical analysis, and the construction of a co-expression network. We focused on 268 WNT annotated signaling genes that are derived from the molecular signature database.

Results: Using the scenarios we identified seven prognostic markers for overall survival and event-free survival. Three genes are novel prognostic markers; two with favorable outcome (PSMD2, PPARD) and one with unfavorable outcome (XPNPEP). The remaining four genes (LEF1, SFRP2, RUNX1, and AXIN2) were previously identified but we could refine the patient groups. Three AML risk groups were further analyzed and the co-expression network showed that only the good risk group harbors frequent promoter hypermethylation and significantly correlated interactions with proteasome family members.
\end{abstract}

Conclusion: Our results provide novel insights in WNT signaling in AML, we discovered new and previously identified prognostic markers and a refinement of the patient groups.

\section{Background}

The development of stem cells and progenitor cells into mature functioning cells depends on, among others, the correct functioning of the WNT signaling pathway [1,2]. As an example, Wnt3a deficiency leads in leukemia cells to reduced numbers of long-term hematopoietic stem

\footnotetext{
* Correspondence: e.taskesen@tudelft.nl

'Delft Bioinformatics Lab (DBL), Delft University of Technology, 2628CD Delft, the Netherlands

Full list of author information is available at the end of the article
}

cells (HSC) and multipotent progenitors [3,4]. In Acute Myeloid Leukemia (AML), the WNT pathway genes do not show frequent genetic alterations but rather gene silencing by promoter methylation [5-7]. The epigenetic alterations plays a critical role in initiation, progression, and maintenance of the disease phenotype. Promoter methylation of genes in the WNT pathway (e.g., AXIN2, sFRP1, sFRP2, sFRP4, sFRP5, DKK1, DKK3, APC, RUNX1, SOX17, WIF1, RASSF1A, LKB1/STK11, cyclin D1, TCF1, $L E F 1$. CTNNB1) causes genes to be downregulated in 
their mRNA expression, thereby deregulation of the WNT signaling. The relation between these genetic and epigenetic factors is especially important when studying the disease phenotype, such as for patients with deregulated WNT signaling. This is also shown in previous studies that reported associations of promoter methylation of WNT signaling genes with adverse prognosis $[6,8]$.

Over the past years, genetic and epigenetic studies have, independently from each other, provided important new insights in the underlying pathology of Acute Myeloid Leukemia (AML) [9-11]. AML is a heterogeneous disease that can roughly be classified in three risk groups; good, intermediate and the poor risk group. The good risk group consists of patients with a translocation between chromosome 15 and $17(\mathrm{t}(15 ; 17))$ or a translocation between chromosomes 8 and $21(t(8 ; 21)$ or an inversion of chromosome $16(\operatorname{inv}(16))$. The intermediate risk group consists patients with a normal karyotype, and the poor risk group with a complex karyotype (patients more than 3 cytogenetic abnormalities). Previous studies analysed genetic and epigenetic changes of WNT signaling genes mostly in patients with intermediate risk [6], however, DNA hypermethylation was found to be enriched for the good risk group [12]. These studies however analysed a limited number of genes in the WNT signaling pathway. A comprehensive analysis of the relationships between DNA-methylation and transcript expression by means of all annotated genes of the WNT signaling pathway has not been done.

For this study we developed a statistical approach to model the mRNA transcript expression with DNAmethylation abundance and applied it to a complete list of WNT signaling genes. This allowed us to refine groups of patients from which genes have specific promoter methylation (either hypermethylated, hypomethylated or not methylated at all), and gene expression (either up regulated, down regulated or not changed in expression), potentially important for better clinical diagnostics. Further we provided novel insights of WNT signaling in AML that may inspire therapeutic interventions. Our integration strategy provides useful biological interpretation for the detected prognostic markers and for patient clustering.

\section{Results \\ Analysis of prognostic markers by means of gene-state scenarios}

It has previously been shown that DNA-methylation or gene expression levels of genes can be used as predictive features to determine clinical outcome of AML patients $[6,8]$. We speculated that patient groups can be refined when DNA-methylation and gene expression levels of genes are analysed in an integrated fashion. We therefore categorized each gene in one of nine scenarios, each representing a unique combination of up/down/no gene expression and hyper/hypo/no methylation of its promoter region (see Methods and Figure 1A). We next assessed whether a state of a gene is predictive for clinical outcome for Overall Survival (OS) and Event-Free Survival (EFS) using univariate (log-rank test) and multivariate analyses (Cox's proportional hazard ratio model) (See method section). We used in total 344 AML patients and marked a gene as a prognostic marker if both the univariate and multivariate analysis resulted in significant differences in clinical outcome with $\mathrm{P} \leq 0.05$ (See method section and Additional file 1).

\section{Re-analysis of known WNT associated prognostic markers for leukemia}

We started analysing thirteen WNT signaling genes that are known to have important function in leukemia, i.e., sFRP1, sFRP2, sFRP4, DKK1, DKK3, APC, APC2, RUNX1, WIF1, TCF1, LEF1, LKB1/STK11, and CTNNB1, and could confirm on our data that LEF1 [13], SFRP2 [6], RUNX1 [14], and AXIN2 have prognostic value.

\section{AXIN2}

AXIN inhibition protein 2 (AXIN2) is a well-established readout for WNT signaling activation across various species and has been used within the hematopoietic system in reporter mice as readout of WNT activation[15]. We detected that the expression levels of AXIN2 separates patients in two groups for which unfavourable outcome is seen for patients with significant downregulated AXIN2 expression levels in OS (Figure 2G, Table 1) and EFS (Additional file 2G, and 3). Note that this is irrespective of the DNA-methylation levels in the promoter region of AXIN2 (i.e., AXIN2's genes-state is in scenario 8). Surprisingly, this is in contrast to solid tumours, for instance colon carcinomas, in which high WNT signaling is a risk factor.

LEF1

For lymphoid enhancer-binding factor-1 (LEF1) it was shown that high gene expression levels are associated with favourable clinical outcome in CN-AML [13], and thus that downregulated expression levels are then associated with unfavourable clinical outcome. We indeed detected in our data that patients with significant downregulated LEF1 mRNA expression levels have an unfavourable clinical outcome for OS (Figure 2A, Table 1), and EFS (Additional file $2 \mathrm{~A}$, and 3 ). In addition, the unfavourable clinical outcome was only seen for patients that showed no significant differences of promoter DNA-methylation of LEF1 (i.e., patients with the gene-state of $L E F 1$ in scenario 8). In fact, from the 97 patients with significant downregulated gene expression levels of $L E F 1$, only 61 patients with $L E F 1$ in scenario 8 could be marked with significant unfavourable clinical outcome whereas the other 36 patients (i.e., $L E F 1$ in scenario 5) could not. 


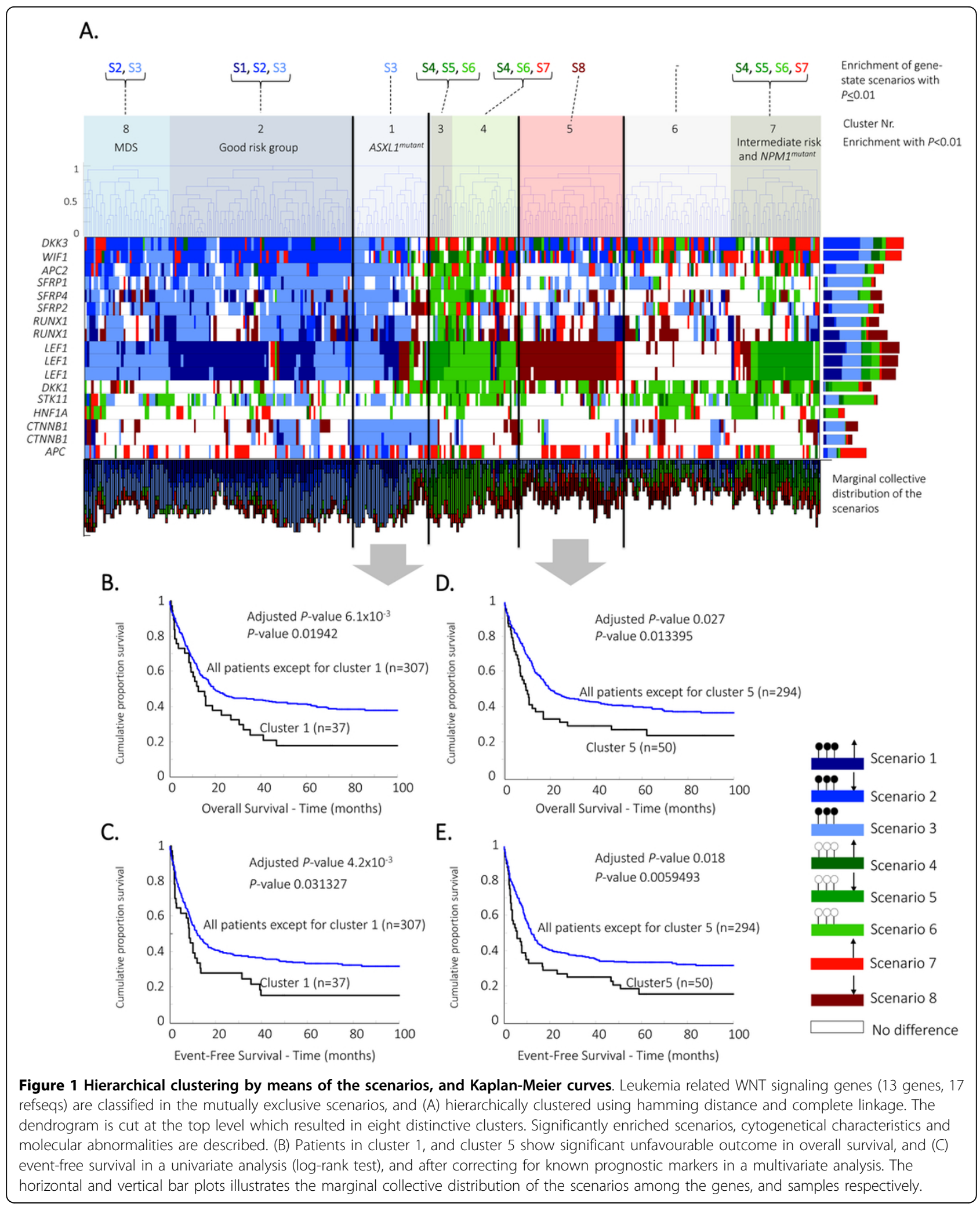


A.

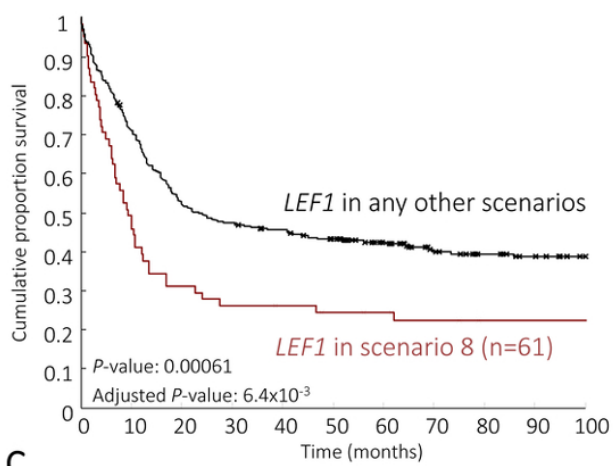

C.
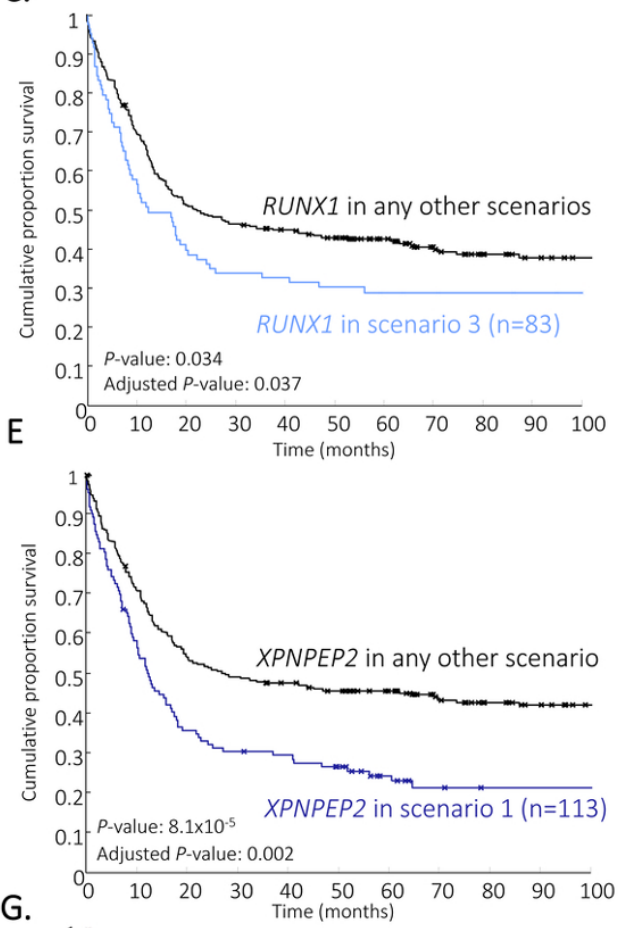

G.

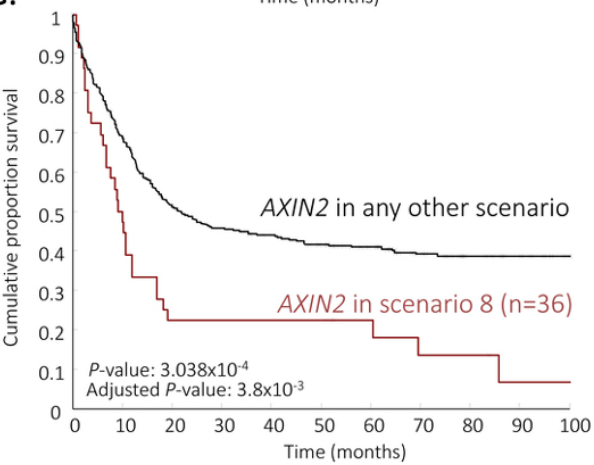

B.
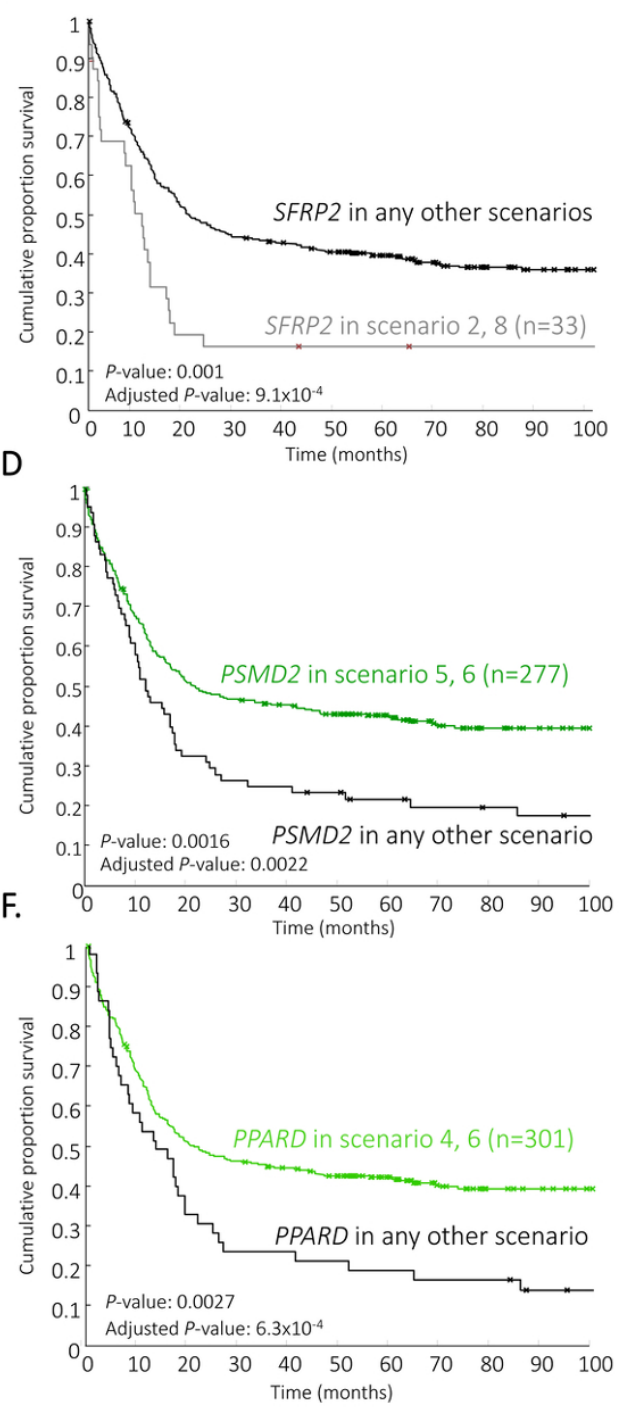

Figure 2 Kaplan-Meier curves for the identified prognostic genes. The Kaplan-Meier curves for overall survival illustrates the identified prognostic markers and the patient groups based on the classification of gene scenario. (A) LEF1, (B) SFRP2, (C) RUNX1, (D) PSMD2, (E) XPNPEP2, (F) PPARD, and (G) AXIN2. Significance for each prognostic marker is computed by comparing patients in the particular scenario versus patients in any other scenario (black line) using a univariate (log-rank test, indicated by the P-value), and multivariate analysis (Cox's proportional hazard ratio model, indicated by the adjusted $P$-value). 
Table 1 Multivariate analysis for the identified prognostic genes for overall survival (OS)

\begin{tabular}{|c|c|c|c|c|}
\hline Variables & $P$-value & $\mathrm{HR}$ & 95\% Cl-low & Cl-high \\
\hline \multicolumn{5}{|l|}{ Overall survival } \\
\hline LEF1 (scenario 8) & 0.00642 & 1.58 & 1.14 & 2.19 \\
\hline CEBPA $A^{\mathrm{dm} \alpha}$ & 0.00214 & 0.33 & 0.16 & 0.67 \\
\hline$F L T 3^{I T D} \beta$ & 0.00025 & 1.77 & 1.30 & 2.40 \\
\hline$N P M 1^{+\beta}$ & 0.00005 & 0.51 & 0.37 & 0.71 \\
\hline WBC count ${ }^{\delta}, \times 10^{9} / \mathrm{L}$ & 0.00886 & 1.00 & 1.00 & 1.00 \\
\hline $\mathrm{Age}^{\$}$ & 0.00005 & 1.02 & 1.01 & 1.03 \\
\hline \multicolumn{5}{|l|}{ Overall survival } \\
\hline SFRP2 (scenario 2, 8) & 0.00091 & 2.00 & 1.33 & 3.00 \\
\hline CEBPA $A^{\mathrm{dm} \alpha}$ & 0.00160 & 0.32 & 0.16 & 0.65 \\
\hline$F L T 3^{I T D} \beta$ & 0.00007 & 1.87 & 1.37 & 2.55 \\
\hline$N P M 1^{+\beta}$ & 0.00006 & 0.52 & 0.38 & 0.71 \\
\hline WBC count ${ }^{\delta}, \times 10^{9} / \mathrm{L}$ & 0.00470 & 1.00 & 1.00 & 1.01 \\
\hline $\mathrm{Age}^{\$}$ & 0.00007 & 1.02 & 1.01 & 1.03 \\
\hline \multicolumn{5}{|l|}{ Overall survival } \\
\hline RUNX1 (scenario 3) & 0.03768 & 1.38 & 1.02 & 1.88 \\
\hline CEBPA $A^{\mathrm{dm} \alpha}$ & 0.00308 & 0.34 & 0.17 & 0.69 \\
\hline$F L T 3^{I T D} \beta$ & 0.00023 & 1.78 & 1.31 & 2.41 \\
\hline$N P M 1^{+\beta}$ & 0.00004 & 0.51 & 0.37 & 0.71 \\
\hline WBC count ${ }^{\delta}, \times 10^{9} / \mathrm{L}$ & 0.01106 & 1.00 & 1.00 & 1.00 \\
\hline $\mathrm{Age}^{\$}$ & 0.00001 & 1.02 & 1.01 & 1.03 \\
\hline \multicolumn{5}{|l|}{ Overall survival } \\
\hline PSMD2 (scenario 5, 6) & 0.00220 & 0.60 & 0.43 & 0.83 \\
\hline CEBPA $A^{\mathrm{dm} \alpha}$ & 0.00130 & 0.31 & 0.15 & 0.63 \\
\hline$F L T 3^{I T D} \beta$ & 0.00006 & 1.87 & 1.38 & 2.55 \\
\hline$N P M 1^{+\beta}$ & 0.00160 & 0.59 & 0.42 & 0.82 \\
\hline WBC count ${ }^{\delta}, \times 10^{9} / \mathrm{L}$ & 0.00270 & 1.00 & 1.00 & 1.01 \\
\hline $\mathrm{Age}^{\$}$ & 0.00009 & 1.02 & 1.01 & 1.03 \\
\hline \multicolumn{5}{|l|}{ Overall survival } \\
\hline XPNPEP (scenario 1) & 0.00200 & 1.56 & 1.18 & 2.07 \\
\hline CEBPA $A^{\mathrm{dm} \alpha}$ & 0.00084 & 0.30 & 0.15 & 0.60 \\
\hline$F L T 3^{I T D} \beta$ & 0.00130 & 1.66 & 1.22 & 2.26 \\
\hline$N P M 1^{+\beta}$ & 0.00020 & 0.55 & 0.40 & 0.75 \\
\hline WBC count ${ }^{\delta}, \times 10^{9} / \mathrm{L}$ & 0.01190 & 1.00 & 1.00 & 1.00 \\
\hline $\mathrm{Age}^{\$}$ & 0.00015 & 1.02 & 1.01 & 1.03 \\
\hline \multicolumn{5}{|l|}{ Overall survival } \\
\hline PPARD (scenario 4, 6) & 0.00063 & 0.52 & 0.36 & 0.76 \\
\hline CEBPA $^{\mathrm{dm} \alpha}$ & 0.00075 & 0.29 & 0.14 & 0.60 \\
\hline$F L T 3^{I T D} \beta$ & 0.00011 & 1.83 & 1.35 & 2.48 \\
\hline$N P M 1^{+\beta}$ & 0.00084 & 0.57 & 0.41 & 0.79 \\
\hline WBC count ${ }^{\delta}, \times 10^{9} / \mathrm{L}$ & 0.00360 & 1.00 & 1.00 & 1.01 \\
\hline $\mathrm{Age}^{\$}$ & 0.00001 & 1.02 & 1.01 & 1.03 \\
\hline \multicolumn{5}{|l|}{ Overall survival } \\
\hline AXIN2 (scenario 8) & 0.0038 & 1.79 & 1.21 & 2.66 \\
\hline CEBPA $^{\mathrm{dm} \alpha}$ & 0.0009 & 0.30 & 0.15 & 0.61 \\
\hline$F L T 3^{I T D} \beta$ & 0.0003 & 1.77 & 1.30 & 2.40 \\
\hline$N P M 1^{+\beta}$ & 0.0003 & 0.55 & 0.40 & 0.76 \\
\hline WBC count $\delta, \times 10^{9} / \mathrm{L}$ & 0.0100 & 1.00 & 1.00 & 1.00 \\
\hline $\mathrm{Age}^{\$}$ & 0.00004 & 1.02 & 1.01 & 1.03 \\
\hline
\end{tabular}

Cox proportional hazard model for multivariable analyses of prognostic markers for overall survival. Analyses included 344 AML patients. Abbreviations: HR, hazard ratio; $\mathrm{Cl}$, confidence interval; $\alpha$ CEBPA ${ }^{\text {double-mutation }}$ status versus CEBPA ${ }^{\text {wt }}, \beta F L T 3^{\text {ITD }}$ versus no FLT3 $3^{\text {ITD }}$ mutation, $\beta$ NPM1 ${ }^{\text {mutant }}$ versus $N P M 1^{\text {wt }}, \delta$ WBC count higher than $20 \times 10^{9} / L$ versus lower than $20 \times 10^{9} / L$, \$ Age is used as continuous variable. 


\section{SFRP2}

For the secreted frizzled-related protein 2 (SFRP2) it has previously been shown that DNA hypermethylation of the promoter region is associated with unfavourable clinical outcome [6]. In our data, we also detected significant unfavourable clinical outcome for patients with promoter hypermethylated SFRP2 (Figure 2B) but these patients also showed downregulated expression levels of SFRP2 (i.e., patients with SFRP2 in scenario 2). In addition to that, unfavourable clinical outcome was also seen for patients with solely downregulated expression levels of SFRP2 (i.e., patients with SFRP2 in scenario 8). These two patients groups were therefore combined in our approach, as described in the method section (OS: Figure 2B, Table 1 and EFS: Additional file 2B, and 3).

\section{RUNX1}

Runt-Related Transcription Factor is a tumor suppressor in myeloid neoplasms, and mutations in $R U N X 1$ are previously associated with unfavourable outcome in $\mathrm{CN}$ AMLs $[14,16]$. In addition, it was previously demonstrated that inhibition of $R U N X 1$ activity could be a promising therapeutic strategy for AMLs with leukemogenic fusion proteins [17].We detected significant unfavourable clinical outcome in OS (Figure 2C, Table 1), but not for EFS (Additional file 2C, and 3), for patients with DNA hypermethylation but without significant differences of gene expression levels of RUNX1 (i.e., patients with RUNX1 in scenario 3). Thus we show that not all patients with DNA hypermethylation have poor survival; it also depends on the gene expression levels.

Taken together, by re-analysing known WNT associated markers, we could re-establish the prognostic role of a number of these markers in AML patients, but we also illustrate that patient groups could be refined by using an integrative analysis of gene expression and DNA-methylation profiles. A comparison of our results with the use of only gene expression profiles, we detected that only SFRP2 showed significant associations with OS and EFS, i.e., downregulated expression levels (scenario 2, 5 and 8) are associated with unfavourable clinical outcome compared to the upregulated expression levels (scenario 1, 4 and 7, additional file 4A, B). The use of solely DNA-methylation profiles did not result in significant associations with clinical outcome, i.e., hypermethylation (scenario 1, 2 and 3) versus hypomethylation (scenario 4, 5, 6).

\section{Detection of novel WNT associated prognostic markers for leukemia}

In addition to the WNT signaling genes that are known to have important functions in leukemia, we also analysed the 268 WNT signaling genes annotated in the molecular signature database (see Methods). We speculated that novel prognostic markers could be identified by using the refined gene-state scenarios. Three additional genes
(PSMD2, XPNPEP2, and PPARD) showed significant differences in OS and EFS.

\section{PSMD2}

The proteasome non-catalytic subunit is previously identified as a potential therapeutic target in lung adenocarcinomas [18]. For PSMD2 we detected significant favourable OS (Figure 2D, Table 1) and EFS (Additional file $2 \mathrm{D}$, and 3 ), for patients with a promoter hypomethylation and downregulated gene expression levels (i.e., patients with the PSMD2 gene-state in scenario 5). In addition, favourable clinical outcome was also seen for patients with solely promoter hypomethylation (i.e., patients with the PSMD2 gene-state in scenario 6). These two patients groups were therefore combined in our approach.

\section{XPNPEP2}

Patients were detected with significant unfavourable clinical outcome for OS (Figure 2E, Table 1) and EFS (Additional file 2E, and 3) if the promoter of gene $\mathrm{X}$ Prolyl Amino peptidase 2 was DNA hypermethylation and the expression was upregulated (i.e., patients with $X P N P E P 2$ in scenario 1 ).

\section{PPARD}

The third independent prognostic marker that we detected is Peroxisome Proliferator-Activated Receptor Delta $(P P A R D)$. We detected significant favourable clinical outcome for OS (Figure 2F, Table 1) and EFS (Additional file 2F, and 3), among patients with hypomethylated levels and without significant change of gene expression levels (i.e., patients with XPNPEP2 in scenario 6). In addition, favourable clinical outcome was also seen for patients with promoter hypomethylation and upregulated gene expression levels (i.e., patients with the PSMD2 gene-state in scenario 4). These two patients groups could therefore be combined.

Taken together, by analysing methylation and expression data together we could associate three more WNT signaling genes with prognostic relevance, enforcing the role of WNT signaling in leukemia. A comparison of the integrative results with the use of only gene expression profiles revealed that only XPNPEP2 was significantly association with OS and EFS in univariate and multivariate analysis, i.e., upregulated expression levels (combined scenario 1, 4 and 7) are associated with unfavourable clinical outcome compared to the upregulated expression levels (combined scenario 2, 5 and 8, additional file $4 \mathrm{C}, \mathrm{D})$. On the other hand, the use of solely DNA-methylation profiles showed significant associations with clinical outcome for PSMD2 (additional file 4E, F), and PPARD (additional file $4 \mathrm{G}, \mathrm{H}$ ) by a comparison of hypermethylation (combined scenario 1, 2 and 3) versus hypomethylation (combined scenario 4, 5, 6). For both cases, promoter hypermethylation is associated with unfavourable outcome. 
Hierarchical clustering of AML patients based on genestate scenarios revealed grouping of patients that are associated with clinical outcome

It has been shown previously that patients with two or more hypermethylated WNT genes are associated with unfavourable survival compared to patients with zero or one hypermethylated WNT genes [8]. We therefore asked ourselves whether we can improve the subtyping of patients based on the patterns of multiple WNT signaling genes. We hierarchically clustered the patients, using the 13 WNT signaling genes that are previously associated with leukemia, with a hamming distance over the genestate description and complete linkage (Figure 1A). Eight clusters are detected in total from which four clusters showed enrichment with known cytogenetic or molecular abnormalities, and three patient clusters showed enrichment with DNA hypermethylation. We focussed only on cluster 1 and 5 as these showed significant differences in clinical outcome for both OS and EFS compared to the patients in any of the other clusters.

\section{Cluster 1}

Cluster 1 is one of the three clusters (together with cluster 2 , and 8) that showed significant overrepresentation of genes with DNA hypermethylation. However, only cluster 1 resulted in significant unfavourable clinical outcome for OS and EFS (Figure 1B, C and Additional file 5). The two other clusters are enriched for the good cytogenetical risk group (cluster 2), and for MDS patients (cluster 8). DNA hypermethylation of WNT signaling genes among the good cytogenetical risk group [8], and MDS/secondary AMLs $[5,19]$ are supported by previous results. In fact, it has also been illustrated that patients with multiple hypermethylated WNT signaling genes are associated with unfavourable clinical outcome [8]. But we demonstrate that significant unfavourable clinical outcome is only seen for patients with overrepresentation of genes in scenario 3 (DNA hypermethylation without significant change of gene expression levels). Patients in this cluster (cluster 1) also showed significant occurrence of ASXL1 mutations, which has previously been described to be a prognostic marker for unfavourable outcome [20]. To determine whether the DNA hypermethylation (genes in scenario 3) causes the unfavourable outcome of cluster 1 patients or whether it is caused by the $A S X L 1$ mutations, we included $A S X L 1$ as an additional covariate in the multivariate analysis. With a result that Cluster 1 still showed significant survival for OS $(P<0.025$, HR: 1.58$)$ and EFS $(P<0.028$, HR: 1.57), and thereby indicating that patients with DNA hypermethylation of WNT genes are associated with unfavourable survival.

\section{Cluster 5}

Patients in cluster 5 also showed unfavourable OS and EFS (Figure 1D, E). These patients were enriched for genes with their state in scenario 8 but showed no enrichment for other cytogenetical or molecular abnormalities.

With the cluster analysis we illustrate the complex behaviour of multiple WNT signaling genes and their combined association with clinical outcome. Using both the methylation and expression profiling we are able to refine current subtyping of AML patient's giving new leads to further research.

\section{The good risk patients group is characterized by DNA hypermethylation of the WNT pathway}

We analysed the gene expression and DNA-methylation profiles of the 268 WNT signaling genes to infer whether WNT signaling genes could explain differences among the risk groups. We detected that DNA hypermethylation (genes whose state is in the scenarios 1,2 , or 3) is significantly more seen in the WNT genes among the good risk group patients when compared to the intermediate and poor risk group (Figure 3A-C, blue shaded colours). By looking into the gene-states of the WNT genes, we found that $33 \%$ of the WNT genes are in similar gene-states for all the three risk groups, whereas only 9 genes (10 refseqs) showed unique genestates for the risk groups (Figure 3D). Among them 'proteasome 26S subunit, non-ATPase, 10' (PSMD10). For PSMD10 there is evidence that positive expression levels associate with short survival time of patients in pancreatic ductal adenocarcinoma tissues [21]. This is in line with our results where the poor risk group is detected with overexpression, and the good and intermediate risk group with promoter DNA hypermethylation of PSMD10.

\section{Good risk patients group shows interactions between WNT signaling genes and genes of the proteasome network}

To gain more insights in the role of DNA-methylation for the signaling cascade of WNT we examined whether the methylation status associates with the functional importance of a gene. To get a notion of the functional importance of a gene, we assumed that the degree of a gene, in a co-expression network is indicative for its role: i.e., a gene with frequent interactions potentially regulates (or is regulated by) more genes. Consequently, we construct a co-expression network by means of pairwise Pearson correlations between the continuous mRNA expression levels for the poor, intermediate and good AML risk group (see methods).

The good risk group contained in total 167 significantly expressed genes for which 65 are upregulated and 102 downregulated. Pairwise correlation resulted in 96 unique genes with one or more significant pairwise interactions (Figure 4A). DNA hypermethylation was 



Figure 3 The distribution of gene scenarios, and gene specificity among the three AML risk groups. Classification of the 268 WNT signaling genes into the mutually exclusive scenarios for (A) good risk group, (B) intermediate risk group, and (C) poor risk group. (D) Genes with unique gene-state scenario among the AML risk groups.

detected in $36 \%$ of the genes, whereas the genes with highest degree consists of DNA hypermethylated genes, such as KREMEN1, APC2, CSTF1, NFATC2, SPAG8, WIF1 (Figure 4A).

The intermediate risk group contains 160 significantly regulated genes (63 upregulated, and 97 downregulated), for which 67 unique genes had significant pairwise interactions. Nine genes are detected with DNA hypermethylation from which KREMEN1, and SPAG8 are listed with the highest degree (Figure 4B). The poor risk group contains 56 significantly upregulated and 89 downregulated genes for which 68 unique genes had significant pairwise interactions. The genes with the highest degree contained, among others, the DNA hypermethylated gene SPAG8 (Figure 4C).

Interestingly, genes with a high degree appear to be DNA hypermethylation, such as for gene KREMEN1, which is seen in similar state in the good and intermediate risk group but not in the poor risk group. KREMEN1 encodes a high-affinity dickkopf homolog 1 (DKK1) transmembrane receptor that functionally cooperates with $D K K 1$ to block wingless (WNT)/beta-catenin signaling. Another finding is that each risk group contains two similar sub-networks (i.e., network 1 and 2, Figure 4), indicating that genes behave, to some extent similar. The first network consists of genes such as KREMEN1, SPAG8, $D K K 3$, and NFATC2 that appeared to be under control of DNA hypermethylation in the good risk group, but not for the intermediate and poor risk group. The second network is significantly enriched for proteasome gene family members (PSM) which provides instructions for making parts of the proteasomes, and are part of the cell's quality control system. Remarkably, the good risk group has frequent co-regulatory effects with the proteasome network whereas this seems absent in the intermediate and poor risk group. To summarize, we show that DNA hypermethylated genes appear to be highly connected in the co-expression network, and interact with a proteasome network for the good risk group, which is not the case for the intermediate and poor risk group.

\section{Conclusions}

We created a computational approach to classify genes into scenarios that describe, in a discrete manner, the state of a gene considering its gene expression and the DNAmethylation levels of its promoter region. Throughout the whole study we used these scenarios to analyse WNT signaling genes for patients with Acute Myeloid Leukemia. As in previous studies [6], we also detected high frequency of DNA hypermethylation of WNT genes in AML but could now better characterize patients by jointly analysing the DNA-methylation and gene expression profiles.

We illustrate that the integration of DNA-methylation and gene expression into scenarios can refine and detect novel prognostic gene markers. This is especially important when groups are selected using prognostic markers 
A.



B.

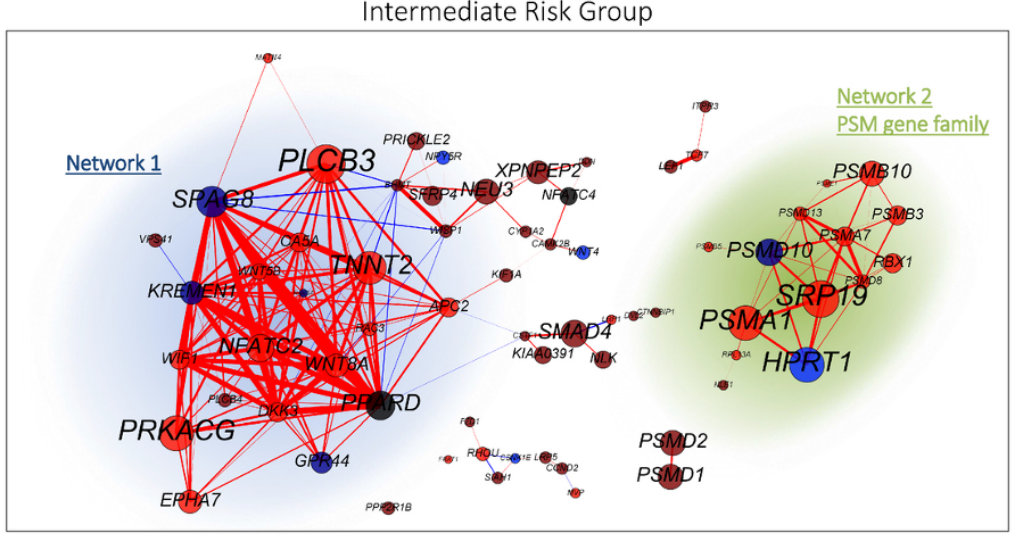

C.

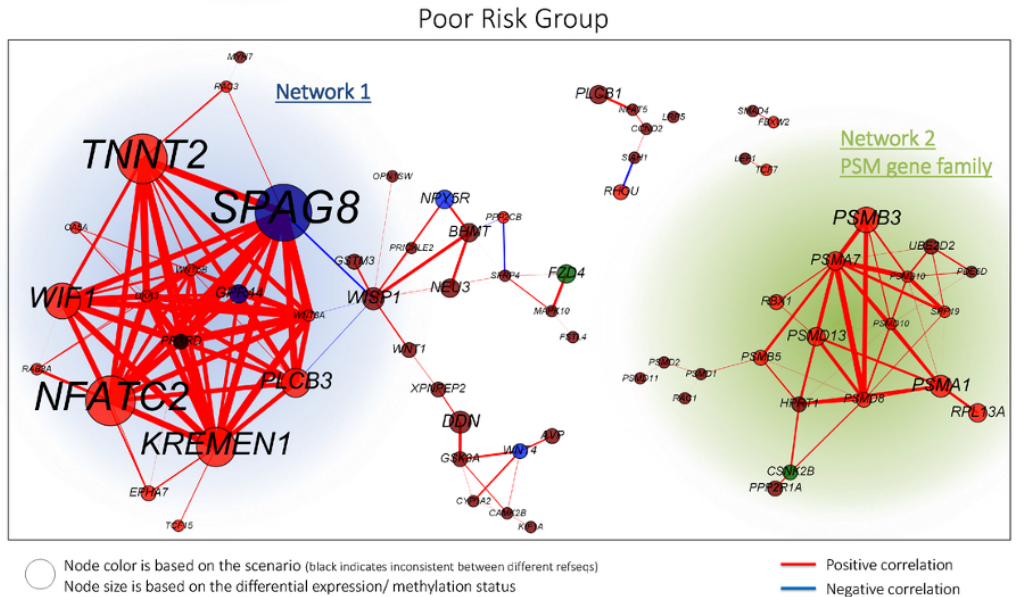

Figure 4 Co-expression network among cytogenetical risk groups. Significant expressed genes (comparison with CD34+ normal samples) among each risk group are pairwise correlated using the continuous gene expression levels. A network is subsequently build by means of the significant pairwise correlations. The colour of the nodes (genes) illustrates the scenarios, whereas the size of the node and the label text is based on the - $\log 10$ ( $P$-value) for differential expression or methylation. Edges with positive correlation are indicated in red, whereas negative correlations are indicated in blue. The thickness of the edges are based on the absolute correlation measure which varies between 0.6 and 1. The proteasome gene family network (PSM-family) describes members of the proteasome complex. 
for targeted drug therapy. Using our integrative approach we detected in total seven independent prognostic markers from which two (SFRP2 and XPNPEP2) overlapped if only gene expression profiles are used (out of nine). Based on only DNA-methylation profiles, 14 prognostic markers are detected from which two (PSMD2 and $P P A R D$ ) overlapped with our integrative approach (Additional file 6).

Our approach also refined patient groups for which previously was shown that multiple hypermethylated WNT genes were associated with unfavourable survival [8]. We demonstrate that unfavourable survival of patients is associated with promoter hypermethylation without change of gene expression levels (scenario 3). In addition, we detected that good risk group patients significantly harbour DNA hypermethylation (which is in line with previously results [12], but we provide a mechanistic understanding of the functional role of DNA hypermethylation by examining the co-expression gene networks. Although the exact functional relationships of DNA-methylation and the proteasome is difficult to comprehend, the importance of proteasome inhibitors is described for Multiple Myeloma, where the use of proteasome inhibitors are potentially interesting as they can serve as therapeutic strategy by directly targeting both the tumor and bone disease [22] Their role in AML is, however, less known. Yet, proteasome inhibition is recently introduced as a promising novel anticancer therapy for AML [23]. Further, the proteasome inhibitor Bortezomib sensitizes AML with myelomonocytic/monocytic phenotype (M4/M5 AML based on FAB classification) in AML cell lines [24].

Although many studies illustrate that gene expression or DNA-methylation levels in isolation can serve as a prognostic marker, our computational approach proved effective in the identification of prognostic markers and improves characterization of patient groups by the integration of gene expression and DNA-methylation profiles. This is especially important when groups are selected on prognostic markers for targeted drug therapy.

\section{Methods}

\section{Patient samples}

Diagnostic bone marrow (BM) or peripheral blood (PB) samples from 344 adults were analysed. All patients provided written informed consent in accordance with the Declaration of Helsinki. All trials were approved by the Institutional Review Board of Erasmus University Medical Center. Summary of clinical, cytogenetical and molecular features of the patient are previously described [9].

\section{Genome-wide high throughput data}

Two high throughput datasets were used in this study: genome-wide mRNA expression profiling data (GEP) and for the same 344 samples, genome-wide DNA-methylation profiling data (DMP). In addition, eight GEP and eleven DMP reference samples (CD34+) are available. These two assays provide mRNA expression and promoter DNAmethylation abundance levels. GEP data is generated using Affymetrix HGU133 plus2.0 (Santa Clara, CA, USA). Normalization of raw GEP data was processed with RMA $[25,26]$. In addition, probes are remapped to refseq transcripts using a custom CDF definition. Gene expression levels are mean centered over all samples. DMP-data is generated using the HELP assay and pre-processed as described previously [27]. Both data sets are annotated using UCSC hg19, and are available at the NCBI Gene Expression Omnibus [accession numbers GSE14468 and GSE18700]. Refseq transcripts from GEP and probesets from DMP are included on the following terms: 1) Probeset are annotated with chromosome information, genomic location and strand position; 2) Mapping of probesets was without known sequence variation; 3) Annotated genes for the refseq transcripts and probesets overlapped between both data sets. This resulted into 12925 uniquely overlapping genes for 14292 refseq transcripts (GEP) and 19899 probesets (DMP). However, in this study we were specifically interested in the genes that are annotated as being part of the WNT signaling pathway.

\section{WNT pathway signaling genes are derived from the Molecular Signature database}

Eight WNT associated pathways are utilized from the Molecular Signature Database (MSigDB, v3.0), and we derived 364 unique genes from them: Biocarta WNT Pathway (26 genes), KEGG WNT Signaling Pathway (151 genes), Morf WNT1 (101 genes), Reactome Signaling by WNT ( 58 genes), ST WNT Beta Catenin Pathway (31 genes), ST WNT CA2 Cyclic GMP Pathway (19 genes), Willert WNT Signaling (20 genes), WNT Signaling (89 genes). From the 364 WNT associated genes, 268 were available in the gene expression profiling data (291 refseqs), and overlapped with the DNA-methylation profiling data (539 probesets).

\section{Discretization of DNA-methylation and mRNA expression profiles into gene-state scenarios for individual cancer samples}

Discretization of the data was used to categorize each gene (refseq) into one of nine mutually exclusive scenarios. A scenario describes in a discrete manner the change in gene expression (upregulation, downregulation or no regulation) and of the same gene, the change in DNA-methylation level (hypermethylation, hypomethylation or no methylation). Classification of genes in one of the scenarios was accomplished by first discretising the continuous gene expression profiles in comparison to $\mathrm{CD} 34+$ control samples (Additional file 1A,B). A gene transcript (refseq) is marked as upregulated/downregulated if the mRNA 
expression levels were lower/higher than the 99\% confidence interval of the CD34+ normal bone marrow samples of the same gene transcript. Otherwise, a gene transcript is marked as no difference in mRNA expression. Similarly, the continuous DNA-methylation profiles were also discretized in comparison to CD34+ normal bone marrow samples (Additional file 1A,B). A probeset is marked as hypomethylated/hypermethylated if the DNA-methylation levels were higher/lower than the $99 \%$ confidence interval of the CD34+ normal bone marrow samples of the same probeset. Otherwise, a gene is marked as no DNA-methylation. Both discretized data sets are subsequently integrated to define the gene status in terms of scenarios (Additional file 1C). There are nine possible scenarios in total. The first three scenarios [1-3] describe DNA hypermethylation with mRNA transcript expression (up, down or no/low expression), and are colored in different shades of blue. Scenarios [4-6], describe DNA hypomethylation with mRNA transcript expression (up, down or no/low expression), and are colored in different shades of green. Scenarios $[7,8]$ describe upregulation and downregulation but without difference in DNA-methylation levels, and are colored in different shades of red. The ninth scenario contains genes for which no difference in gene expression and no difference in DNA-methylation levels were observed, or cases for which the gene promoters contained multiple probesets with inconsistent DNA-methylation levels (e.g., one probeset with hypermethylated whereas another with hypomethylated, independent of the gene expression level). We will denote this scenario as no difference, and is colored as white in the figures. The colors are consistently used throughout the whole manuscript.

\section{Discretization of DNA-methylation and mRNA expression} profiles into gene-state scenarios for patient risk groups Discretization and integration of gene expression and DNA-methylation data of the cytogenetical risk groups (poor, intermediate and good) into gene-state scenarios is performed in two steps. The first step involves comparison of the GEP patient data with GEP reference samples (CD34+), and the DMP patient data with DMP reference (CD34+) data by means of a student T-test. Significant genes $($ FDR $<0.01)$ with a significant positive $\mathrm{T}$-statistic are categorized as upregulated/hypomethylated and with a significant negative T-statistic as downregulated/hypermethylated. For DNA-methylation probesets, the absolute fold difference must also be $\geq 1.5$ compared to the reference samples in order to have a sufficient effect-size. The second step is the integration of the GEP and DMP gene status in terms of scenarios. This is similar as described for the individual cancer samples, thus each gene is classified into one of the nine scenarios based on the DNA-methylation with mRNA transcript expression level changes.

\section{Construction of the co-expression networks}

To construct the co-expression network and determine the gene-degree, we applied the following approach: 1) gene expression levels among the AML risk groups must be significantly upregulated or downregulated compared to the CD34+ normal samples (as described in the method section); 2) pairwise Pearson correlations among the continuous mRNA gene expression levels are computed between the significantly detected WNT signaling genes; 3) gene interactions with $\mathrm{r}>0.6$ and $P<$ 0.05 are marked, and used in the network; 4) the degree for each gene is determined by the number of edges it contains; 5) each gene in the network is illustrated in terms of the mutually exclusive scenarios.

\section{Survival analysis based on gene-state scenarios}

To assess whether a gene is significantly associated with clinical outcome, we compared patient groups based on their gene-state scenarios (Additional file 1D). To prevent high multiple test correction by comparing a maximum of nine patient groups (i.e., patients that are categorized in each of the nine scenarios), we first clustered patient groups on survival. We applied the following approach: 1) for each gene a pairwise comparison between patient groups (e.g., among the gene-state scenarios) is performed using the log-rank test (for OS or EFS) (Additional file 1E). The pairwise log-rank test is then used as a distance matrix (indicating the similarity between patient groups survival) which is in turn hierarchically clustered using Euclidean distance and average linkage. The dendrogram is then cut horizontally through the leaves at the smallest height that results in two clusters (Additional file 1F). The resulting two clusters, each containing one or multiple patient groups are subsequently compared against each other for its difference in OS and EFS using univariate (log-rank test) and multivariate analyses (Cox's proportional hazard ratio model) (Additional file 1G). For the multivariate analysis we adjusted for the covariates; age, white blood cell count, CEBPA double-mutation,$N P M 1^{\text {mutation }}$, and FLT3 $3^{I T D}$ status. Note that the definition of survival endpoints were based on the recommended consensus criteria [28].

\section{Additional material}

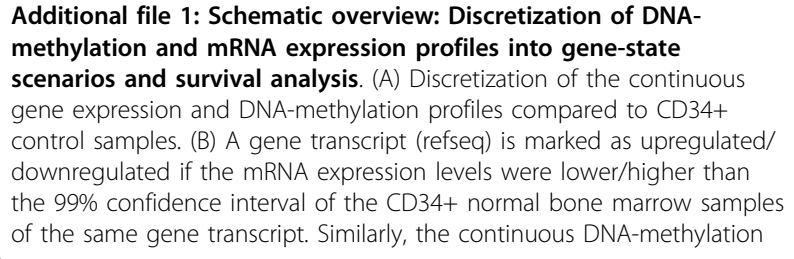


profiles were also discretized in comparison to CD34+ normal bone marrow samples. (C) Both discretized data sets are subsequently integrated to define the gene status (scenarios) in terms of mRNA transcript expression and DNA hypermethylation. (D) Grouping of samples that have the same gene-state scenario. In this example, Gene 1 groups patients in scenario S1, S2, S4, S7 and S8. (E) Pairwise comparison between patient groups using the log-rank test (for OS or EFS). (F) The distance matrix based on the pairwise log-rank test is hierarchically clustered, and cut horizontally through the leaves that results in two clusters. (G) The resulting two clusters, each containing one or multiple patient groups are subsequently compared against each other for its difference in OS and EFS using univariate (log-rank test) and multivariate analyses (Cox's proportional hazard ratio model).

Additional file 2: Kaplan-Meier curves for the identified prognostic genes for Event-Free Survival. The Kaplan-Meier curves illustrates the prognostic markers and the patient groups based on the classification of gene scenario. (A) LEF1, (B) SFRP2, (C) RUNX1, (D) PSMD2, (E) XPNPEP2, (F) $P P A R D$, and (G) AXIN2. Significance for each prognostic marker is computed by comparing patients in the particular scenario versus patients in any other scenario (black line) using a univariate (depicted by the $P$-value), and multivariate analysis (depicted by the adjusted $P$-value).

Additional file 3: Multivariate analysis for the identified prognostic genes for Event-Free Survival (EFS). Cox proportional hazard model for multivariable analyses of prognostic markers for Event-free survival. Analyses included 344 AML patients. Abbreviations: HR, hazard ratio; Cl, confidence interval; $\alpha$ CEBPA double-mutation status versus CEBPA ${ }^{w t}, \beta F L T 3^{\text {ITD }}$ versus no FLT3 ${ }^{\text {TID }}$ mutation, $\beta$ NPM1 $1^{\text {mutant }}$ versus NPM1 $1^{\text {wt }}, \delta$ WBC count higher than $20 \times 10^{9} / \mathrm{L}$ versus lower than $20 \times 10^{9} / \mathrm{L}$, \$ Age is used as continuous variable.

\section{Additional file 4: Kaplan-Meier curves by using solely gene} expression or DNA-methylation profiles. The Kaplan-Meier curves illustrates the prognostic markers and the patient groups based on the comparison upregulated (scenario 1,4,7) versus downregulated (scenario $2,5,8)$ gene expression levels, and hypermethylated (scenario 1,2,3) versus hypomethylated (scenario $4,5,6)$ levels. Significance is assessed by comparing patients using a univariate (depicted by the $P$-value), and multivariate analysis (depicted by the adjusted $P$-value).

Additional file 5: Multivariate analysis for cluster 1 and cluster 5 for OS and EFS. Cox proportional hazard model for multivariable analyses of cluster 1 and cluster 5 for OS and EFS. Abbreviations: HR, hazard ratio; $\mathrm{Cl}$, confidence interval; $\alpha$ CEBPA $^{\text {double-mutation }}$ status versus CEBPA ${ }^{\text {wt }}, \beta$ FLT3 $^{\text {TID }}$ versus no FLT3 $3^{I T D}$ mutation, $\beta$ NPM1 $1^{\text {mutant }}$ versus NPM1 $1^{\text {wt }}, \delta$ WBC count higher than $20 \times 10^{9} / \mathrm{L}$ versus lower than $20 \times 10^{9} / \mathrm{L}$, $\$$ Age is used as continuous variable.

Additional file 6: Venn diagram illustrating the overlap of prognostic markers detected by the integrative approach, the use of solely gene expression profiles, and solely DNA-methylation profiles. Using the integrative approach, seven prognostic markers are detected. Using solely gene expression profiles, 9 prognostic markers are detected. Using solely DNA-methylation profiles, 14 prognostic markers are detected.

\section{Competing interests}

The authors declare that they have no competing interests.

\section{Authors' contributions}

ET implemented the methodologies, analysed, interpreted the data, and drafted the manuscript. ET, FJTS, and MJTR participated in the design of the study and contributed to the writing of the paper. All authors provided relevant input at different stages of the project, and read and approved the final manuscript.

\section{Acknowledgements}

The authors thank Jeroen de Ridder for the discussions.

\section{Declarations}

This work is part of the BioRange program of the Netherlands Bioinformatics Centre (NBIC), which is supported by the Netherlands Genomics Initiative (NGI).
This article has been published as part of BMC Bioinformatics Volume 16 Supplement 4, 2015: Selected articles from the 9th IAPR conference on Pattern Recognition in Bioinformatics. The full contents of the supplement are available online at http://www.biomedcentral.com/bmcbioinformatics/ supplements/16/S4.

\section{Authors' details}

${ }^{1}$ Delft Bioinformatics Lab (DBL), Delft University of Technology, 2628CD Delft, the Netherlands. ${ }^{2}$ Netherlands Bioinformatics Centre (NBIC), the Netherlands. ${ }^{3}$ Department of Immunohematology and Bloodtransfusion, 2300RC Leiden, the Netherlands.

\section{Published: 23 February 2015}

\section{References}

1. Luis TC, Ichii M, Brugman MH, Kincade P, Staal FJ: Wnt signaling strength regulates normal hematopoiesis and its deregulation is involved in leukemia development. Leukemia 2012, 26(3):414-21.

2. Kikuchi A: Tumor formation by genetic mutations in the components of the wnt signaling pathway. Cancer Sci 2003, 94(3):225-9.

3. Staal FJ, Luis TC, Tiemessen MM: Wnt signalling in the immune system: Wnt is spreading its wings. Nat Rev Immunol 2008, 8(8):581-93.

4. Luis TC, Weerkamp F, Naber BA, Baert MR, de Haas EF, Nikolic T, Heuvelmans S, De Krijger RR, van Dongen JJ, Staal FJ: Wnt3a deficiency irreversibly impairs hematopoietic stem cell self-renewal and leads to defects in progenitor cell differentiation. Blood 2009, 113(3):546-54.

5. Figueroa ME, Skrabanek L, Li Y, Jiemjit A, Fandy TE, Paietta E, Fernandez H, Tallman MS, Greally JM, Carraway H, Licht JD, Gore SD, Melnick A: Mds and secondary aml display unique patterns and abundance of aberrant dna methylation. Blood 2009, 114(16):3448-58.

6. Griffiths EA, Gore SD, Hooker C, McDevitt MA, Karp JE, Smith BD, Mohammad HP, Ye Y, Herman JG, Carraway HE: Acute myeloid leukemia is characterized by wnt pathway inhibitor promoter hypermethylation. Leuk Lymphoma 2010, 51(9):1711-9.

7. Martin V, Valencia A, Agirre X, Cervera J, San Jose-Eneriz E, Vilas-Zornoza A, Rodriguez-Otero P, Sanz MA, Herrera C, Torres A, Prosper F, RomanGomez J: Epigenetic regulation of the non-canonical wnt pathway in acute myeloid leukemia. Cancer Sci 2010, 101(2):425-32.

8. Valencia A, Roman-Gomez J, Cervera J, Such E, Barragan E, Bolufer P, Moscardo F, Sanz GF, Sanz MA: Wnt signaling pathway is epigenetically regulated by methylation of wnt antagonists in acute myeloid leukemia. Leukemia 2009, 23(9):1658-66.

9. Figueroa ME, Lugthart S, Li Y, Erpelinck-Verschueren C, Deng X, Christos PJ, Schifano E, Booth J, van Putten W, Skrabanek L, Campagne F, Mazumdar M, Greally JM, Valk PJ, Lowenberg B, Delwel R, Melnick A: Dna methylation signatures identify biologically distinct subtypes in acute myeloid leukemia. Cancer Cell 2010, 17(1):13-27.

10. Valk PJ, Verhaak RG, Beijen MA, Erpelinck CA, Barjesteh van Waalwijk van Doorn-Khosrovani S, Boer JM, Beverloo HB, Moorhouse MJ, van der Spek PJ, Lowenberg B, Delwel R: Prognostically useful gene-expression profiles in acute myeloid leukemia. N Engl J Med 2004, 350(16):1617-28.

11. Lee $E$, Jung $H$, Radivojac $P$, Kim JW, Lee D: Analysis of aml genes in dysregulated molecular networks. BMC Bioinformatics 2009, 10(Suppl 9):2

12. Hou HA, Kuo YY, Liu CY, Lee MC, Tang JL, Chen CY, Chou WC, Huang CF, Lee FY, Liu MC, Yao M, Tien HF: Distinct association between aberrant methylation of wnt inhibitors and genetic alterations in acute myeloid leukaemia. Br J Cancer 2011, 105(12):1927-33.

13. Metzeler KH, Heilmeier B, Edmaier KE, Rawat VP, Dufour A, Dohner K, Feuring-Buske M, Braess J, Spiekermann K, Buchner T, Sauerland MC, Dohner H, Hiddemann W, Bohlander SK, Schlenk RF, Bullinger L, Buske C: High expression of lymphoid enhancer-binding factor-1 (lef1) is a novel favorable prognostic factor in cytogenetically normal acute myeloid leukemia. Blood 2012, 120(10):2118-26.

14. Mendler JH, Maharry K, Radmacher MD, Mrozek K, Becker H, Metzeler KH, Schwind S, Whitman SP, Khalife J, Kohlschmidt J, Nicolet D, Powell BL, Carter TH, Wetzler M, Moore JO, Kolitz JE, Baer MR, Carroll AJ, Larson RA, Caligiuri MA, Marcucci G, Bloomfield CD: Runx1 mutations are associated with poor outcome in younger and older patients with cytogenetically normal acute myeloid leukemia and with distinct gene and microrna expression signatures. J Clin Oncol 2012, 30(25):3109-18. 
15. Luis TC, Naber BA, Roozen PP, Brugman MH, de Haas EF, Ghazvini M, Fibbe WE, van Dongen JJ, Fodde R, Staal FJ: Canonical wnt signaling regulates hematopoiesis in a dosage-dependent fashion. Cell Stem Cell 2011, 9(4):345-56.

16. Greif PA, Konstandin NP, Metzeler KH, Herold T, Pasalic Z, Ksienzyk B, Dufour A, Schneider F, Schneider S, Kakadia PM, Braess J, Sauerland MC, Berdel WE, Buchner T, Woermann BJ, Hiddemann W, Spiekermann K, Bohlander SK: Runx 1 mutations in cytogenetically normal acute myeloid leukemia are associated with a poor prognosis and up-regulation of lymphoid genes. Haematologica 2012, 97(12):1909-15.

17. Goyama S, Schibler J, Cunningham L, Zhang Y, Rao Y, Nishimoto N, Nakagawa M, Olsson A, Wunderlich M, Link KA, Mizukawa B, Grimes HL, Kurokawa M, Liu PP, Huang G, Mulloy JC: Transcription factor runx1 promotes survival of acute myeloid leukemia cells. J Clin Invest 2013, 123(11):4979.

18. Matsuyama Y, Suzuki M, Arima C, Huang QM, Tomida S, Takeuchi T, Sugiyama R, Itoh Y, Yatabe Y, Goto H, Takahashi T: Proteasomal noncatalytic subunit psmd2 as a potential therapeutic target in association with various clinicopathologic features in lung adenocarcinomas. $\mathrm{Mol}$ Carcinog 2011, 50(4):301-9.

19. Wang $H$, Fan R, Wang XQ, Wu DP, Lin GW, Xu Y, Li WY: Methylation of wnt antagonist genes: a useful prognostic marker for myelodysplastic syndrome. Ann Hematol 2013, 92(2):199-209.

20. Pratcorona M, Abbas S, Sanders MA, Koenders JE, Kavelaars FG, ErpelinckVerschueren CA, Zeilemakers A, Lowenberg B, Valk PJ: Acquired mutations in asxl1 in acute myeloid leukemia: prevalence and prognostic value. Haematologica 2012, 97(3):388-92.

21. Pfeffer : The study on the expression and significance of survivin and psmd10 in pancreatic ductal adenocarcinoma. GlobeThesis, (2012-05-16).

22. Claire M, Edwards GRM: Proteasome inhibitors and the wnt signaling pathway in myeloma bone disease. Humana Press, Current Clinical Oncology 2010, 211-229.

23. Colado E, Alvarez-Fernandez S, Maiso P, Martin-Sanchez J, Vidriales MB, Garayoa M, Ocio EM, Montero JC, Pandiella A, San Miguel JF: The effect of the proteasome inhibitor bortezomib on acute myeloid leukemia cells and drug resistance associated with the $\mathrm{cd} 34+$ immature phenotype. Haematologica 2008, 93(1):57-66.

24. Dijk M, Murphy E, Morrell R, Knapper S, O'Dwyer M, Samali A, Szegezdi E: The proteasome inhibitor bortezomib sensitizes aml with myelomonocytic differentiation to trail mediated apoptosis. Cancers (Basel) 2011, 3(1):1329-50.

25. Bolstad BM, Irizarry RA, Astrand M, Speed TP: A comparison of normalization methods for high density oligonucleotide array data based on variance and bias. Bioinformatics 2003, 19(2):185-93.

26. Irizarry RA, Hobbs B, Collin F, Beazer-Barclay YD, Antonellis $\mathrm{K}$, Scherf $\mathrm{U}$, Speed TP: Exploration, normalization, and summaries of high density oligonucleotide array probe level data. Biostatistics 2003, 4(2):249-64.

27. Figueroa ME, Melnick A, Greally JM: Genome-wide determination of dna methylation by hpa ii tiny fragment enrichment by ligation-mediated pcr (help) for the study of acute leukemias. Methods Mol Biol 2009, 538:395-407.

28. Dohner H, Estey EH, Amadori S, Appelbaum FR, Buchner T, Burnett AK, Dombret H, Fenaux P, Grimwade D, Larson RA, et al: Diagnosis and management of acute myeloid leukemia in adults: recommendations from an international expert panel, on behalf of the european leukemianet. Blood 2010, 115(3):453-474.

doi:10.1186/1471-2105-16-S4-S4.3d

Cite this article as: Taskesen et al: An integrated approach of gene expression and DNA-methylation profiles of WNT signaling genes uncovers novel prognostic markers in Acute Myeloid Leukemia. BMC Bioinformatics 2015 16(Suppl 4):S4.

\section{Submit your next manuscript to BioMed Central and take full advantage of:}

- Convenient online submission

- Thorough peer review

- No space constraints or color figure charges

- Immediate publication on acceptance

- Inclusion in PubMed, CAS, Scopus and Google Scholar

- Research which is freely available for redistribution

Submit your manuscript at www.biomedcentral.com/submit
Biomed Central 